\title{
Espondilite Anquilosante
}

\section{Ankylosing Spondylitis}

\author{
Anna Lídia Mol Ferreira ${ }^{(1)}$, Corina Quental de Menezes Alvarenga ${ }^{(2)}$, \\ Guilherme de Freitas Barcelos ${ }^{(2)}$, Elizandra Tomazela Laurenti Polito ${ }^{(3)}$
}

A espondilite anquilosante (EA), doença inflamatória crônica que atinge até $1 \%$ da população, pode associar-se a importante limitação funcional e comprometimento da qualidade de vida dos pacientes. Os avanços no conhecimento de sua fisiopatologia e o conseqüente surgimento de novas opções de tratamento - notadamente os agentes biológicos anti-TNF - vêm mudando este cenário. O desenvolvimento de novos conceitos e a disseminação destas informações permitem o diagnóstico mais precoce e a abordagem terapêutica direcionada é capaz de modificar o curso natural da doença. Nesta seção, os autores descrevem estudos recentes que discutem novas diretrizes para o diagnóstico e o tratamento dos pacientes com espondiloartropatias.

Rudwaleit M, Khan MA, Spiecer J: The challenge of diagnosis and classification in early ankylosing spondylitis. Do we need new criteria? (O desafio do diagnóstico e classificação em espondilite anquilosante inicial. Nós precisamos de novos critérios?). Arthritis Rheum 52:1000-85, 2007. Campus Benjamin Franklin, Berlim, Alemanha.

Uma crítica recorrente aos critérios de classificação da espondilite anquilosante (EA) empregados até o momento (critério de Roma, critério de New York e critério modificado de New York) é que tanto alterações de mobilidade espinhal quanto sacroiliíte radiográfica são reflexo do tempo de duração da doença e, portanto, ocorrem em fases mais avançadas. A verificação de sacroiliíte em radiografias convencionais deve ser considerada, sim, marcador de cronicidade e gravidade, e não critério diagnóstico. Notadamente, pelos avanços no conhecimento da fisiopatologia e as novas alternativas de tratamento, que têm impacto importante na evolução da doença, é desejável um diagnóstico o mais precoce possível. Neste artigo, os autores sugerem que pacientes com espondiloartropatia e predomínio de doença axial sejam considerados como tendo espondiloartropatia axial (SpA), independente da presença de sacroiliíte radiográfica, reservando o termo espondilite anquilosante para pacientes com anquilose. Este grupo propõe, então, algoritmo diagnóstico fundamentado em parâmetros clínicos, laboratoriais e de imagem, ponderados por meio de razão de verossimilhança (likelihood ratio LR, Figura 1). Observou-se que em pacientes com lombalgia crônica três ou quatro parâmetros necessitam estar presentes para a probabilidade diagnóstica em torno de 90\% (LR 200), caindo para $80 \%$ no caso de três parâmetros (LR 80) e para $50 \%$ quando presentes apenas dois (LR 20). A ressonância nuclear magnética (RNM) foi introduzida como ferramenta de detecção precoce de inflamação osteoarticular (fase "préradiográfica"). Este modelo ainda não foi validado e não está difundido na prática clínica. No entanto, trata-se de discussão pertinente e deve ser levada em consideração para otimizar a abordagem dos pacientes com espondiloartropatia.

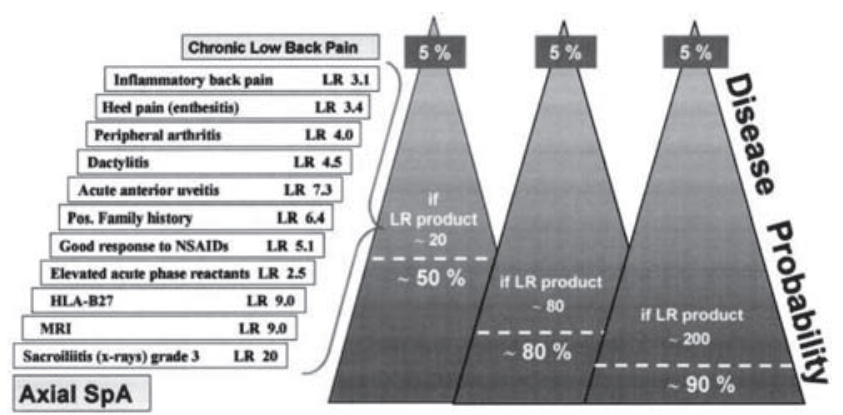

Figura 1 - Esta figura descreve os parâmetros clínicos, radiológicos e laboratoriais a serem avaliados em pacientes com dor lombar crônica e seu respectivo "peso" (LR). 0 produto da multiplicação dos LR dos diferentes parâmetros se associa à probabilidade de tratar-se de SpA (se o produto é cerca de 20, a probabilidade de tratar-se de SpA é cerca de 50\%. Se o produto é cerca de 80 , a probabilidade de SpA é cerca de $80 \%$. Se o LR é $\geq 200$, a probabilidade de SpA alcança $90 \%$ ).

1 Médica residente de Reumatologia do Hospital Santa Casa de Belo Horizonte.

2 Médicos especializandos de Reumatologia do Hospital Santa Casa de Belo Horizonte.

3 Coordenadora do Ambulatório de Espondiloartropatias do Hospital Santa Casa de Belo Horizonte.

Endereço para correspondência: Elizandra T. L. Polito, Rua Domingos Vieira, 587, sala 404 - 30150-240 - Belo Horizonte, MG, e-mail: eliz.polito@gmail.com 
Sieper J, Appel H, Braun J, Rudwaleit M: Critical appraisal of assessment of structural damage in ankylosing spondylitis: Implications for treatment outcomes (Apreciação crítica de avaliação de lesão estrutural em espondilite anquilosante: implicações sobre resultado de tratamentos). Arthritis Rheum 58: 649-56, 2008. Campus Benjamin Franklin e Centro de Pesquisa Reumatológica Alemã, Berlim, Alemanha.

Neste artigo de revisão, os autores discutem as implicações da fisiopatologia da doença e seu tratamento atual na progressão radiológica da espondilite anquilosante (EA). Antiinflamatórios não-esteróides (AINE) e agentes anti-TNF são as únicas formas de tratamento com eficácia comprovada nas manifestações axiais da EA. Os agentes anti-TNF demonstraram importante efeito na redução de sinais/sintomas da doença, melhora funcional e da qualidade de vida, redução de provas de fase aguda e melhora da inflamação osteoarticular mensurada por técnicas de imagem, como ressonância nuclear magnética. No entanto, ao contrário do que acontece na artrite reumatóide (AR), estudos de até dois anos falharam em demonstrar efeito positivo desta classe de drogas na progressão radiológica da doença, mensurada primariamente pela neoformação óssea e progressão dos sindesmófitos. Por outro lado, outros estudos puderam confirmar que pacientes com EA em uso regular de AINE apresentam menor progressão radiológica. Como uma classe de droga com efeitos tão marcantes na apresentação clínica dos pacientes com EA, como os anti-TNF, não têm efeito no dano radiológico atribuído à doença? Os autores discutem diferentes aspectos desta questão. Inicialmente, o parâmetro utilizado para tal mensuração - o stoke ankylosing spondylitis spine score (mSASSS $)$ - tem limitações. Ainda que esta escala contemple erosões osteoarticulares, a proliferação óssea tem maior peso em seu resultado. Outro aspecto importante é o fato de a progressão radiológica ser mais lenta em EA e estudos de dois anos podem não ser o suficiente para sua melhor avaliação.

Os autores discutem também aspectos peculiares da fisiopatologia da doença. Tanto em AR quanto em EA, a inflamação osteoarticular se associa a destruição da cartilagem e erosões ósseas. No entanto, estas ocorrem em sítios diferentes e na EA, após a redução da inflamação, existem evidências de que, em uma tentativa de reparo, o sítio do dano estrutural seja preenchido por tecido fibroso e, a seguir, sofra ossificação, expressa radiologicamente como sindesmófito. Boa parte do efeito positivo dos agentes antiTNF em AR se dá pela redução da atividade osteoclástica. No contexto desta discussão, o achado recente de que a inflamação inibe a atividade osteoblástica é de grande interesse. Este efeito é mediado principalmente pelo TNF e por uma molécula reguladora da via Wnt - o Dkkl - que não sofreria influência dos bloqueadores do TNF. Os autores postulam que os agentes anti-TNF teriam importante efeito na supressão da inflamação, mas não exerceriam qualquer influência na proliferação óssea. Por outro lado, o efeito positivo dos AINEs na progressão radiológica da EA poderia ser explicado por inibição da atividade osteoblástica por meio do bloqueio das prostaglandinas.

Em conclusão, os autores entendem que, para prevenção da progressão radiológica, notadamente de seu componente de neoformação óssea, seria necessária a supressão da inflamação em fases bem precoces, antes da ocorrência de dano da cartilagem e erosões ósseas. Ainda que tenham alguma ligação, a inflamação e a neoformação óssea parecem ser processos primariamente independentes na EA, provavelmente determinados por suscetibilidade genética distinta. Nesta linha de raciocínio, é plausível assumir que a abordagem terapêutica ótima de pacientes com EA deva incluir drogas com efeito supressor da inflamação (por exemplo, anti-TNF) e também agentes capazes de inibir a neoformação óssea (por exemplo, AINEs). Também pela peculiaridade de progressão mais arrastada, os autores acreditam que estudos longitudinais com maior tempo de observação sejam necessários para melhor apreciação destes conceitos.

Lambert RWG, Salonen D, Rahman P, Inman RD, Wong RL, Einstein SG, Thomson GTD, Beaulieu A, Choquette D, Maksymowych WP: Adalimumab significantly reduces both spinal and sacroiliac joint inflammation in patients with ankylosing spondylitis (Adalimumabe reduz significativamente inflamação espinhal e sacroilíaca em pacientes com espondilite anquilosante). Arthritis Rheum 56(12):4005-14, 2007. Universidade de Alberta, Edmonton, Canadá.

Este estudo foi realizado com objetivo de comparar a eficácia do adalimumabe versus o placebo na redução da inflamação sacroilíaca (SI) e espinhal pela imagem de ressonância nuclear magnética (RNM) em pacientes com 
espondilite anquilosante (EA). Foi um estudo multicêntrico, randomizado, duplo-cego, placebo controlado, no qual 82 pacientes com doença ativa foram randomizados para receber adalimumabe $(\mathrm{n}=38)$ e placebo $(\mathrm{n}=44)$. A RNM foi realizada no início, com 12 e 52 semanas. A intensidade da inflamação foi mensurada pela escala de Spondyloarthritis Research Consortium of Canada (SPARCC). Esta escala tem alto grau de reprodutibilidade entre radiologistas e sensibilidade para inflamação. $\mathrm{Na} \mathrm{12}{ }^{\mathrm{a}}$ semana, os pacientes tratados com adalimumabe mostraram significante redução na escala SPARCC de inflamação axial (redução 53,6\%) em comparação com o placebo (aumento 9,4\%) e esta redução se manteve até a $52^{\text {a }}$ semana. $\mathrm{Na} 24^{\mathrm{a}}$ semana, os pacientes que receberam placebo passaram a receber adalimumabe e a redução da inflamação na $52^{\text {a }}$ semana foi semelhante ao grupo adalimumabe. Similar redução na escala SPARCC no esqueleto axial e na SI foi notada mesmo em pacientes que não preencheram os critérios do ASAS (ASsessment in Ankylosing Spondylitis) - não-respondedores, provavelmente porque o ASAS considera a subjetividade do paciente. Esse estudo demonstrou que adalimumabe induz rapidamente a quase completa remissão na inflamação espinhal e SI em 12 semanas, efeito mantido por, pelo meno, um ano em pacientes com EA ativa. Mesmo os que não respondem clinicamente têm redução na inflamação. Se esses achados pela RNM podem predizer a presença ou a ausência de progressão para o dano estrutural, novos estudos são necessários. No entanto, a RNM mostra-se como instrumento importante de avaliação qualitativa da atividade da doença, que pode se somar às medidas clínicas como BASDAI.

Visvanathan S, van der Heijde D, Deodhar A, Wagner C, Baker D.G, Han J, Braun J: Effects of infliximab on markers of inflammation and bone turnover and associations with bone mineral density in patients with ankylosing spondylitis (Efeitos do infliximabe nos marcadores de inflamação e turnover ósseo e associações com densidade mineral óssea em pacientes com espondilite anquilosante). Ann Rheum Dis 2008, publicado on-line, em 21 de maio de 2008. Rheumazentrum Ruhrgebiet, Herne, Alemanha.

Pacientes com espondilite anquilosante (EA) podem apresentar fraturas vertebrais como complicação da doença. Estudos prévios que analisaram os marcadores da formação e reabsorção óssea na EA têm resultados conflitantes. Há como hipótese que as alterações dos marcadores do turnover ósseo após o uso do infliximabe estejam relacionadas à melhora da progressão da doença, evidenciadas pela diminuição da inflamação e aumento da massa óssea. Pacientes com EA $(\mathrm{n}=279)$ foram randomizados para receber placebo ou infliximabe $(5 \mathrm{mg} / \mathrm{kg})$ a cada seis semanas, durante 96 semanas. Na semana 24, o grupo placebo passou a receber infliximabe e a partir da semana 36 o grupo infliximabe poderia ter o aumento da dose até $7,5 \mathrm{mg} / \mathrm{kg}$, se persistissem com BASDAI maior ou igual a três. Foram dosados nas semanas 0, 2, 24 e 102 os marcadores do turnover ósseo - fosfatase alcalina óssea, osteocalcina e telopeptídeo terminal C do colágeno I - e da inflamação - IL-6, VEGF e TGF- $\beta$. A densidade mineral óssea (DMO) do quadril e da coluna lombar foi avaliada na semana 0,24 e 102 . Na semana 24 , os pacientes tratados com infliximabe mostraram aumento significativo da DMO na coluna lombar $(2,5 \%, \mathrm{p}<0,001)$ e no quadril $(0,5 \%$, $\mathrm{p}=0,033)$, comparados com aqueles do grupo placebo
$(0,5 \%$ e $0,2 \%$, respectivamente $)$. Na semana 24 , os pacientes do grupo placebo passaram a receber infliximabe. $\mathrm{Na}$ semana 102, o aumento percentual médio da DMO para coluna lombar e quadril no grupo infliximabe foi de $6,8 \%$ e $1,8 \%$, respectivamente, e no grupo placebo/infliximabe de $4,1 \%$ e $0,9 \%$, respectivamente. Esse aumento foi menos significativo no grupo placebo/infliximabe, sugerindo benefício no tratamento precoce com infliximabe. A presença de sindesmófitos era determinada pela radiografia no início e na 102 semana. Ao contrário do esperado, os pacientes que desenvolveram sindesmófitos tiveram mudanças na DMO semelhantes aos que não os desenvolveram. Aumentos da DMO não foram ligados ao desenvolvimento de sindesmófitos após modelos de regressão múltipla. O grupo em uso do infliximabe teve diminuição dos marcadores inflamatórios, principalmente IL-6 e VEGF, correlacionada ao aumento da DMO. Altos níveis de osteocalcina basal se associaram ao aumento da DMO, sugerindo que esta possa ter valor em predizer o aumento da massa óssea em pacientes com EA tratados com anti-TNF. Em resumo, o uso de infliximabe levou ao aumento significativo na $\mathrm{DMO}$ em pacientes com EA, relacionado à redução dos marcadores da inflamação. 
Ozgocmen S, Godekmerdan A, Ozkurt-Zengin F: Acute-phase response, clinical measures and disease activity in ankylosing spondylitis (Reagentes de fase aguda, medidas clínicas e atividade de doença em espondilite anquilosante). Joint Bone Spine 74:249-53, 2007. Faculdade de Medicina, Universidade de Firat, Elazig, Turquia.

Nesse estudo, os autores fizeram várias correlações entre reagentes de fase aguda e perfil de resposta humoral e celular em pacientes com espondilite anquilosante (EA). Foram avaliados 27 pacientes sem uso de drogas modificadoras de doença (DMARDs) ou de antiinflamatórios não-esteróides (AINES) por, pelo menos, dois meses. Esses pacientes foram classificados de acordo com a atividade de doença: se envolvimento periférico presente ou ausente; se ativos ou inativos, segundo os critérios de remissão/remissão parcial do ASAS e BASDAI. Parâmetros clínicos, exames laboratoriais e imunofenotipagem de linfócitos foram analisados. Entre todas as medidas realizadas, apenas a alfa 1 -antitripsina (AAT) correlacionou-se com os parâmetros clínicos escala visual analógica, BASDAI, BASFI e HAQ-S. Elevação de proteína $C$ reativa (PCR) e velocidade de hemossedimentação (VHS) mostraram relação apenas com artrite periférica. $\mathrm{O}$ único parâmetro com significativa relação com remissão de doença foi o menor nível de AAT. Em relação à AAT, novos estudos são necessários para validar sua dosagem como indicador de atividade ou remissão de doença. Esse estudo confirma a fraca relação entre reagentes de fase aguda e atividade clínica axial da EA. Não obstante o pequeno número de pacientes, o fato de estarem livres de medicamentos fortalece os resultados encontrados.

Keat AC, Gaffney K, Gilbert AK, Harris C, Leeder J: Influence of biologic therapy on return to work in people with work disability due to ankylosing spondylitis (Influência da terapia biológica no retorno ao trabalho em pessoas com incapacidade laboral por causa da espondilite anquilosante). Rheumatology 47:481-3, 2008. Departamento de Reumatologia, Hospital Universitário de Norfolk e Norwich, Reino Unido.

Tanto quanto a artrite reumatóide, a espondilite anquilosante (EA) tem impacto significativo na capacidade laborativa dos pacientes. Como conseqüências existem comprometimento da renda e da auto-estima do paciente e custos significativos para a sociedade. Este é um aspecto importante da doença que deve ser contemplado por ocasião da avaliação de estratégias terapêuticas. Com a intenção de avaliar qual é o efeito da terapia com inibidores do fator de necrose tumoral- $\alpha$ (anti-TNF- $\alpha$ ) na capacidade de trabalho dos pacientes com EA, os autores realizaram este estudo. Sessenta e cinco pacientes em uso de anti-TNF- $\alpha$ foram incluídos nesta avaliação. Vinte e quatro $(36,9 \%)$ estavam recebendo infliximabe, $21(32,3 \%)$ etanercepte e $20(30,7 \%)$ adalimumabe. Questionários estruturados sobre o trabalho antes e depois do tratamento, considerando tempo em horas trabalhadas por semana, tipo de atividade exercida, número de licenças médicas, medidas de atividade (BASDAI), função (BASFI) e resposta ao tratamento foram aplicados antes do tratamento, três e 18 meses após o início do agente antiTNF- $\alpha$. Os dados revelaram que um terço dos pacientes era incapaz para o trabalho antes do tratamento e desses, um em cada quatro retornaram para o trabalho após o tratamento. Globalmente, o número de licenças médicas foi reduzido drasticamente e a avaliação subjetiva do paciente (escala visual analógica) sobre o impacto da EA em sua capacidade laborativa caiu de 7,05 para 2,92 após o tratamento. Não obstante o pequeno número de pacientes impedir análise estatística, confirmou-se associação positiva entre tratamento com biológicos e melhora na capacidade laborativa. No entanto, os efeitos a longo prazo, incluindo avaliações de custo-efetividade, devem ser avaliados em estudos com maior número de pacientes e maior tempo de seguimento.

De Vries MK, Wolbink GJ, Stapel SO, de Vrieze H, van Denderen C, Dijkmans BAC, Aarden LA, van der Horst-Bruinsma IE: Decreased clinical response to infliximab in ankylosing spondylitis is correlated with anti-infliximab formation (Diminuição da resposta clínica com infliximabe em espondilite anquilosante está correlacionada com formação de antiinfliximabe). Ann Rheum Dis 66:1252-4, 2007. Universidade Medical Center, Amsterdam, Holanda.

Sabe-se que os inibidores do fator de necrose tumoral alfa (anti-TNF- $\alpha$ ) são muito eficientes em espondilite an- quilosante (EA). No entanto, desconhece-se porque até $30 \%$ dos pacientes falham em responder ou porque alguns 
respondem inicialmente e depois perdem a eficácia ou desenvolvem reações infusionais. A não-responsividade ao infliximabe pode ocorrer por causa da formação de anticorpos contra anti-TNF- $\alpha$, descritos em pacientes com artrite reumatóide e doença de Crohn. Para provar essa teoria foram selecionados 38 pacientes tratados com infliximabe. Níveis séricos de infliximabe e anticorpo antiinfliximabe foram mensurados na semana 0,24 e 52 e correlacionados com resposta clínica por meio do ASAS-20 (ASsessment in Ankylosing Spondylitis - 20\% melhora) e BASDAI. Após 24 semanas, 24 pacientes $(63 \%)$ preenchiam critérios de resposta ASAS-20, com maiores níveis de infliximabe. Dos não-respondedores, em cinco (36\%) foram verificados anticorpos antiinfliximabe. Após 52 semanas, 21 pacientes (53\%) mantiveram critério de resposta pelo ASAS-20, com níveis de infliximabe significativamente maior $(\mathrm{p}=$ $0,001)$ que não-respondedores. Antiinfliximabe também foi detectado significativamente em mais quantidade nos não-respondedores $(\mathrm{p}=0,04)$. A boa resposta clínica do infliximabe na EA foi associada ao nível sérico de infliximabe e à ausência de anticorpos antiinfliximabe. Mesmo após o aumento da dose de infliximabe, os pacientes com menor resposta clínica mantiveram os níveis séricos de infliximabe. Em 75\% destes foram verificados anticorpos antiinfliximabe. Observou-se relação direta entre a formação de antiinfliximabe com pior resposta clínica e maior risco de reações infusionais. Uma forma de prevenir a formação de antiinfliximabe seria a administração conjunta de outra droga imunossupressora, como o metotrexato, mas este raciocínio não foi confirmado em ensaios clínicos.

Coates LC, Cawkwell LS, Ng NWF, Bennett AN, Bryer DJ, Fraser AD, Emery P, Marzo-Ortega H: Real life experience confirms sustained response to long-term biologics and switching in ankylosing spondylitis (Experiência real de vida confirma resposta sustentada a longo prazo de biológicos e substituições em espondilite anquilosante). Rheumatology 47:897900, 2008. Unidade Acadêmica de Doença Musculoesquelética, Universidade de Leeds e Hospital Chapel Allerton, Chapeltown Road, Leeds, Reino Unido.

A eficácia dos inibidores de fator de necrose tumoral- $\alpha$ (anti-TNF- $\alpha$ ) tem sido confirmada em diferentes ensaios clínicos, mas não existem evidências quanto à eficácia a longo prazo na prática clínica. Uma análise retrospectiva foi conduzida em pacientes com espondilite anquilosante (EA) tratados com anti-TNF- $\alpha$ entre 1999-2006. Todos os pacientes que receberam anti-TNF- $\alpha$ por mais que 12 semanas ou que suspenderam uso por eventos adversos foram incluídos. O total de 113 pacientes ( 84 homens, 29 mulheres, idade média de 45 anos, duração média da doença de 16 anos) receberam biológicos em doses usuais (infliximabe $5 \mathrm{mg} / \mathrm{kg}$ a cada 6 semanas, $\mathrm{n}=60$; etanercepte $50 \mathrm{mg}$ por semana, $\mathrm{n}=62$ e adalimumabe $40 \mathrm{mg}$ a cada 14 dias, $\mathrm{n}=4$ ). Os pacientes estavam recebendo agentes biológicos em média por 20 meses. Os valores médios do BASDAI reduziram de 6,57 para 3,12 , BASFI de 6,57 para 4,16 e PCR de $31 \mathrm{~g} / \mathrm{dL}$ para $7 \mathrm{~g} / \mathrm{dL}$. A melhora foi sustentada por 24 meses com nenhuma perda de eficácia. Somente nove pacientes (8\%) sofreram efeitos colaterais levando à suspensão ou à substituição do primeiro agente anti-TNF- $\alpha$. Na $12^{\underline{a}}$ semana, $88 \%$ responderam ao primeiro biológico. Não-resposta primária foi vista em 13 pacientes (infliximabe $\mathrm{n}=10$, etanercepte $\mathrm{n}=3$ ), dos quais seis se recusaram a receber nova droga biológica. Os outros sete mudaram e aceitaram um segundo agente anti-TNF, com resposta satisfatória em seis. No total, 15 pacientes modificaram seu tratamento para um segundo biológico por efeitos colaterais ou por opção individual e $14(93 \%)$ alcançaram resposta significativa e sustentada. Os resultados não dependeram da droga usada, do tempo de duração da doença e do perfil do HLA-B27. Esse trabalho demonstrou que a incidência de não-respostas primária e secundária é muito menor do que em artrite reumatóide (AR). Existe boa tolerância aos agentes antiTNF e resposta sustentada. Em pacientes com EA que não responderam ao primeiro agente anti-TNF, uma segunda opção deve ser tentada, pois existe boa chance de resposta satisfatória e sustentada. 